\title{
Existence of initial data satisfying the constraints for the spherically symmetric Einstein-Vlasov-Maxwell system
}

\author{
P. Noundjeu ${ }^{1}$; N. Noutchegueme ${ }^{1}$; A. D. Rendall ${ }^{2}$ \\ ${ }^{1}$ Department of Mathematics, Faculty of Sciences, University of Yaounde 1, \\ Box 812, Yaounde, Cameroun \\ e-mail: noundjeu@uycdc.uninet.cm, nnoutch@uycdc.uninet.cm \\ ${ }^{2}$ Max-Planck-Institut für Gravitationsphysik, Am Mühlenberg 1, \\ 14476 Golm, Germany \\ e-mail: rendall@aei.mpg.de
}

\begin{abstract}
Using ODE techniques we prove the existence of large classes of initial data satisfying the constraints for the spherically symmetric EinsteinVlasov-Maxwell system. These include data for which the ratio of total charge to total mass is arbitrarily large.
\end{abstract}

\section{Introduction}

The global dynamical behavior of self-gravitating matter is a subject of central importance in general relativity. A form of matter which has particularly nice mathematical properties is collisionless matter, described by the Vlasov equation. It has the advantage that it lacks the tendency observed in certain other models, such as perfect fluids, that solutions of the equations of motion of the matter lose differentiability after a finite time. These singularities of the mathematical model form an obstacle to further analysis and prevent the study of the global dynamical properties of the solutions. Collisionless matter is free from these difficulties and there is a growing literature on global properties of solutions of the Einstein-Vlasov system [1], [8].

In [7, the authors prove the global existence of asymptotically flat solutions of the spherically symmetric Einstein-Vlasov system, with small initial data. That study concerns uncharged particles. We consider, under the same assumption of spherical symmetry, the case where the particles are charged. To describe the full physical situation, we must then couple the previous system to the Maxwell equations that determine the electromagnetic field created by the fast moving charged particles. As will be seen below, that reduces, in the spherically symmetric case, to its electric part. 
It is appropriate at this point to examine the motivation for considering this particular problem. We are not aware that it has any direct astrophysical applications. There are, however, two reasons why the problem is interesting. The first is that it extends the knowledge of the Cauchy problem for systems involving the Vlasov equation and it will be seen that it gives rise to new mathematical features compared to those cases studied up to now. The second is connected with the fact that it would be desirable to extend the work of [7] beyond spherical symmetry. In particular, it would be desirable from a physical point of view to include the phenomenon of rotation. Unfortunately, presently available techniques do not suffice to get away from spherical symmetry. In this situation it is possible to attempt to obtain further intuition by using the analogy between angular momentum and charge, summed up in John Wheeler's statement, 'Charge is a poor man's angular momentum'. Thus we study spherical systems with charge in the hope that this will give us insight into non-spherical systems without charge. This strategy has recently been pursued in the case of a scalar field as matter model, with interesting results [2, 3].

Concerning the Cauchy problem in general relativity, it is well known that in addition to the Einstein evolution equations there are constraint equations which have to be solved. (See e.g. 44.) It is only of interest to consider the problem of evolution, if the problem of constraints on the initial data can be solved. In our specific case, we are led to a difficulty in solving the constraints on the initial data, that has not previously been considered in the literature. Let us first recall the situation in 7 before seeing how it changes in the case of charged particles. In [7, using the assumption of spherical symmetry, the authors look for two metric functions $\lambda$ and $\mu$, that depend only on the time coordinate $t$ and on the radial coordinate $r$, and for the distribution function $f$ of the uncharged particles that depends on $t, r$ and on the 3-velocity $v$ of the particles; the metric functions $\lambda, \mu$ are subject to the Einstein equations with sources generated by the distribution function $f$ of the collisionless uncharged particles which is itself subject to the Vlasov equation. They show that the Einstein equations to determine the unknown metric functions $\lambda$ and $\mu$, turn out to be two first order ODE in the radial variable $r$, coupled to the Vlasov equation in $f$. Putting $t=0$ in the Einstein equations yields two constraint equations that link the three initial data that are the two initial data for $\lambda(t, r)$ and $\mu(t, r)$ denoted $\stackrel{\circ}{\lambda}(r)$ and $\stackrel{\circ}{\mu}(r)$ respectively, and the initial datum for $f(t, r, v)$ denoted by $\stackrel{\circ}{f}(r, v)$. The equations to be solved are two first order ODE for $\stackrel{\circ}{\lambda}, \stackrel{\circ}{\mu}$. The mass function $m(t, r)$ is defined as an integral of $f$ over the hypersurfaces of constant $t$. The exact definition is given in the next section. Provided the initial mass function $\stackrel{\circ}{m}(r)=m(0, r)$ is everwhere less than $r / 2$ the function $\stackrel{\circ}{\lambda}$ can be determined from the relation $1-2 m / r=e^{-2 \lambda}$. Once this has been done $\mu$ can be determined by integration. In this way functions $\stackrel{\circ}{\lambda}$ and $\stackrel{\circ}{\mu}$ can be determined in a straightforward way when $\stackrel{\circ}{f}$ is given, provided the inequality $2 m / r<1$ is satisfied on the initial hypersurface. Thus a simple parametrization of the set of initial data satisfying the constraints is obtained. They can be constructed 
from a non-negative function $f$ which is required to satisfy one inequality.

In the case of charged particles, the problem of constraints on the initial data is not so easy. We consider the case of a spherically symmetric electric field $\vec{E}$ of the form $\vec{E}(t, r)=e(t, r) \frac{\vec{r}}{r}$, where $e(t, r)$ is an unknown scalar function and $\vec{r}$ the position vector in $\mathbb{R}^{3}$. We denote by $\stackrel{\circ}{e}$ the initial data for $e(t, r)$. The Maxwell equations imply a constraint equation on the initial data, that is a first order ODE in the radial variable $r$. We now have to face the problem of constraints between the four initial data $\stackrel{\circ}{\lambda}, \stackrel{\circ}{\mu}, \stackrel{\circ}{f}, \stackrel{\circ}{e}$. If the equations for $\stackrel{\circ}{\lambda}, \stackrel{\circ}{f}$, and $\stackrel{\circ}{e}$ can be solved then $\stackrel{\circ}{\mu}$ can be determined by a simple integration as in the case of uncharged particles. On the other hand the problem of determining the first three quantities is more difficult in the charged case due to the coupling of the gravitational and electromagnetic constraints. Any solution of the constraints must satisfy the condition $2 m / r<1$ on the initial hypersurface. However in the charged case $m$ includes a contribution due to the energy density of the electric field and this depends on $e$ and $\lambda$. As a consequence, in contrast to the uncharged case, this condition cannot be expressed as an inequality on $f$ alone. Moreover, the equation for $e$ also contains $\lambda$ and so it is not possible to solve the electromagnetic constraint separately from the gravitational one. There is no alternative but to solve the two constraints together. When $\stackrel{\circ}{f}$ is prescribed the constraints define a system of two coupled nonlinear ordinary differential equations for $\stackrel{\circ}{\lambda}$ and $\stackrel{\circ}{e}$. These equations are singular at $r=0$.

Solving the ordinary differential equations arising from the constraints is a task which must necessarily be mastered before undertaking the study of the problem of evolution for the Einstein-Vlasov-Maxwell system. Since the equations are singular they cannot be handled by standard results of ODE theory alone. In this paper, we prove, using statements developed in 9] on singular ODE, that, under smallness assumptions on the prescribed initial data function $\circ$, $f$, a corresponding global solution of the constraints exists. This provides a way of showing the existence of initial data $\stackrel{\circ}{\lambda}, \stackrel{\circ}{\mu}, \stackrel{\circ}{e}$, in the case of an asymptotically flat spacetime with a regular center. In other words, we prove that the results obtained for the constraint equations in [7] that focuses on the case of uncharged particles, extend to the case of charged particles, in the sense that the initial datum $\stackrel{\circ}{f}$ can still be considered as the only arbitrary initial datum.

Given the complicated nature of the constraint equations it seems impractible to describe the most general class of functions $\stackrel{\circ}{f}$ for which the constraints can be solved. What we have been able to do is to describe two large classes of functions $\stackrel{\circ}{f}$ for which it is possible. The first case treated is a rather obvious one to try. The charge $q$ of a particle is a free parameter in the equations. In the case $q=0$ the equations reduce to the uncharged situation analysed in [7]. A perturbation argument then allows the case with fixed $\stackrel{\circ}{f}$ and small $q$ to be treated. A more exotic class of initial data is obtained by a rigorous perturbation argument in a regime where the density of particles tends to zero and the charge 
per particle tends to infinity. This allows the construction of initial data which coincide outside a compact set with data for the Reissner-Nordström solution with arbitrarily large charge to mass ratio. The restriction of these data to the region outside a large radius does not allow an electrovacuum interior which has a regular centre or corresponds to a black hole but, as this construction shows, does allow a regular (dynamical) interior with charged matter.

The paper is organized as follows. In Sect. 2, we recall the spherically symmetric Einstein-Vlasov-Maxwell system, from which we deduce the constraint equations. In Sect. 3, we recall the main result of [9] which we use and we prove the existence of large classes of solutions of the constraint equations for the Einstein-Vlasov-Maxwell system. The derivation of some facts required in Sect. 3 concerning regions where there are no particles is contained in an appendix.

\section{Formulation of the problem}

We consider fast moving collisionless particles with unit mass and charge $q$. The basic spacetime is $\left(\mathbb{R}^{4}, g\right)$, with $g$ a Lorentzian metric with signature $(-,+,+,+)$. In what follows, we assume that Greek indices run from 0 to 3 and Latin indices from 1 to 3 , unless otherwise specified. We also adopt the Einstein summation convention. The metric $g$ reads locally, in cartesian coordinates $\left(x^{\alpha}\right)=\left(x^{0}, x^{i}\right) \equiv(t, \tilde{x})$ :

$$
d s^{2}=g_{\alpha \beta} d x^{\alpha} \otimes d x^{\beta}
$$

The assumption of spherical symmetry means that we can take $g$ of the following form (Schwarzschild coordinates)

$$
d s^{2}=-e^{2 \mu} d t^{2}+e^{2 \lambda} d r^{2}+r^{2}\left(d \theta^{2}+\sin ^{2} \theta d \varphi^{2}\right)
$$

where $\mu=\mu(t, r) ; \lambda=\lambda(t, r) ; t \in \mathbb{R} ; r \in[0,+\infty[; \theta \in[0, \pi] ; \varphi \in[0,2 \pi]$. The Einstein-Vlasov-Maxwell system reads:

$$
\begin{gathered}
R_{\alpha \beta}-\frac{1}{2} g_{\alpha \beta} R=8 \pi\left(T_{\alpha \beta}(f)+\tau_{\alpha \beta}(F)\right) \\
\mathcal{L}_{X(F)} f=0 \\
\nabla_{\alpha} F^{\alpha \beta}=J^{\beta} ; \quad \nabla_{\alpha} F_{\beta \gamma}+\nabla_{\beta} F_{\gamma \alpha}+\nabla_{\gamma} F_{\alpha \beta}=0
\end{gathered}
$$

with:

$$
\begin{aligned}
T_{\alpha \beta}(f) & =-\int_{\mathbb{R}^{3}} p_{\alpha} p_{\beta} f \omega_{p} ; \quad \tau_{\alpha \beta}(F)=-\frac{g_{\alpha \beta}}{4} F_{\gamma \nu} F^{\gamma \nu}+F_{\beta \gamma} F_{\alpha}{ }^{\gamma} \\
J^{\beta}(f)(x) & =q \int_{\mathbb{R}^{3}} p^{\beta} f(x, p) \omega_{p}, \quad \omega_{p}=|g|^{\frac{1}{2}} \frac{d p^{1} d p^{2} d p^{3}}{p_{0}}, p_{0}=g_{00} p^{0}, \\
X^{\alpha}(F) & =\left(p^{\alpha},-\Gamma_{\beta \gamma}^{\alpha} p^{\beta} p^{\gamma}-q p^{\beta} F_{\beta}{ }^{\alpha}\right),
\end{aligned}
$$


where $\Gamma_{\beta \gamma}^{\alpha}$ denote the Christoffel symbols. Here, $x=\left(x^{\alpha}\right)$ is the position and $p=$ $\left(p^{\alpha}\right)$ is the 4 -momentum of particles. In the expressions above, $f$ stands for the distribution function of the charged particles, $F$ stands for the electromagnetic field created by the charged particles. Here (2.3) are the Einstein equations for the metric tensor $g=\left(g_{\alpha \beta}\right)$ with sources generated by both $f$ and $F$, that appear in the stress-energy tensor $T_{\alpha \beta}+\tau_{\alpha \beta}$. Equation (2.4) is the Vlasov equation for the distribution function $f$ of the collisionless particles and (2.5) are the Maxwell equations for the electromagnetic field $F$, with source (current) generated by $f$ through $J=J(f)$. One verifies that the conservation laws $\nabla_{\alpha}\left(T^{\alpha \beta}+\tau^{\alpha \beta}\right)=0$ hold if $f$ satisfies the Vlasov equation.

By the assumption of spherical symmetry, we can take $g$ in the form (2.2). One shows, using the Maxwell equation that $F$ reduces to its electric part. We take it in the form $E=\left(E^{\alpha}\right)$ with $E^{0}=0, E^{i}=e(t, r) \frac{x^{i}}{r}$, and then, a straightforward calculation shows that:

$$
\begin{aligned}
\tau_{00} & =\frac{1}{2} e^{2(\lambda+\mu)} e^{2}(t, r) ; \quad \tau_{0 i}=0 \\
\tau_{i j} & =\frac{1}{2} e^{2 \lambda} e^{2}(t, r)\left\{\left(\delta_{i j}-\frac{x_{i} x_{j}}{r^{2}}\right)-e^{2 \lambda} \frac{x_{i} x_{j}}{r^{2}}\right\},
\end{aligned}
$$

where $\delta_{i j}$ is the Kronecker symbol.

These relations and results of [7] show that the spherically symmetric EinsteinVlasov-Maxwell system implies the following first order ODE system in $\lambda, \mu, f$, $e$ :

$$
\begin{gathered}
e^{-2 \lambda}\left(2 r \lambda^{\prime}-1\right)+1=8 \pi r^{2} \rho \\
e^{-2 \lambda}\left(2 r \mu^{\prime}+1\right)-1=8 \pi r^{2} p \\
\frac{\partial f}{\partial t}+e^{\mu-\lambda} \frac{v}{\sqrt{1+v^{2}}} \cdot \frac{\partial f}{\partial \tilde{x}}-\left(e^{\mu-\lambda} \mu^{\prime} \sqrt{1+v^{2}}+\dot{\lambda} \frac{\tilde{x} \cdot v}{r}-q e^{\lambda+\mu} e(t, r)\right) \frac{\tilde{x}}{r} \cdot \frac{\partial f}{\partial v}=0 \\
\frac{d}{d r}\left(r^{2} e^{\lambda} e(t, r)\right)=q r^{2} e^{\lambda} \int_{\mathbb{R}^{3}} f(t, \tilde{x}, v) d v
\end{gathered}
$$

where $\lambda^{\prime}=\frac{\partial \lambda}{\partial r} ; \quad \dot{\lambda}=\frac{\partial \lambda}{\partial t}$ and:

$$
\begin{array}{r}
\rho(t, \tilde{x})=\int_{\mathbb{R}^{3}} f(t, \tilde{x}, v) \sqrt{1+v^{2}} d v+\frac{1}{2} e^{2 \lambda(t, \tilde{x})} e^{2}(t, \tilde{x}) \\
p(t, \tilde{x})=\int_{\mathbb{R}^{3}}\left(\frac{\tilde{x} \cdot v}{r}\right)^{2} f(t, \tilde{x}, v) \frac{d v}{\sqrt{1+v^{2}}}-\frac{1}{2} e^{2 \lambda(t, \tilde{x})} e^{2}(t, \tilde{x})
\end{array}
$$

Here (2.6)-2.7) are the Einstein equations for $\lambda$ and $\mu,(2.8$ is the Vlasov equation for $f$ and (2.9) is the Maxwell equation for $e$. Here $\tilde{x}$ and $v$ belong to $\mathbb{R}^{3}, r:=|\tilde{x}|, \tilde{x} \cdot v$ denotes the usual scalar product of vectors in $\mathbb{R}^{3}$, and 
$v^{2}:=v \cdot v$. The distribution function $f$ is assumed to be invariant under simultaneous rotations of $\tilde{x}$ and $v$, hence $\rho$ and $p$ can be regarded as functions of $t$ and $r$. It is assumed that $f(t)$ has compact support for each fixed $t$. We are interested in asymptotically flat spacetimes which leads to imposing the boundary condition that:

$$
\lim _{r \rightarrow \infty} \mu(t, r)=0
$$

They should also have a regular centre which means that in addition to $\lambda, \mu$ and $e$ being smooth functions of $t$ and $r$, including at $r=0$, the boundary condition $\lambda(t, 0)=0$ should be satisfied for all $t$. Note that the condition that $\lambda(t, r)$ tends to zero as $r \rightarrow \infty$, which is part of asymptotic flatness, follows from the field equations and the fact that $f(t)$ has compact support. This is because in the region where $f$ vanishes the general solution of (2.6) satisfies the condition $\lambda(r)=O(r)$ as $r \rightarrow \infty$.

Let $m(r)=4 \pi \int_{0}^{r} s^{2} \rho(s) d s$. This is the mass function referred to in the introduction. Its limit $M$ as $r \rightarrow \infty$ is the total or ADM mass of the system. The function $n=\int_{\mathbb{R}^{3}} f d v$ is the number density of particles and $n q$ the charge density. The total charge of the system is given by $Q=4 \pi q \int_{0}^{\infty} s^{2} e^{\lambda(s)} n(s) d s$.

Now, define the initial data by:

$$
f(0, \tilde{x}, v)=\stackrel{\circ}{f}(\tilde{x}, v) ; \lambda(0, \tilde{x})=\stackrel{\circ}{\lambda}(\tilde{x})=\stackrel{\circ}{\lambda}(r) ; \stackrel{\circ}{e}(0, \tilde{x})=\stackrel{\circ}{e}(\tilde{x})=\stackrel{\circ}{e}(r)
$$

with $\stackrel{\circ}{f} \in C_{0}^{1}$ being a continuously differentiable function with compact support, which is nonnegative and spherically symmetric, i.e

$$
\forall A \in S O(3), \forall(\tilde{x}, v) \in \mathbb{R}^{6}, \stackrel{\circ}{f}(A \tilde{x}, A v)=\stackrel{\circ}{f}(\tilde{x}, v) .
$$

We obtain the constraint equations on the initial data by taking (2.6), (2.7) and (2.9) for $t=0$, that give:

$$
\begin{aligned}
& e^{-2 \dot{\lambda}^{\prime}}\left(2 r \stackrel{\circ}{\lambda}^{\prime}-1\right)+1=8 \pi r^{2} \int_{\mathbb{R}^{3}} \sqrt{1+v^{2}} \stackrel{\circ}{f}(r, v) d v+4 \pi r^{2} e^{2 \dot{\lambda}^{\circ}} e^{2} \\
& \frac{d}{d r}\left(r^{2} e^{\stackrel{\circ}{\lambda}} \stackrel{\circ}{e}\right)=q r^{2} e^{\stackrel{\circ}{\lambda}} \int_{\mathbb{R}^{3}} \stackrel{\circ}{f}(r, v) d v=J(\stackrel{\circ}{f}) \\
& e^{-2 \stackrel{\circ}{\lambda}}\left(2 r \stackrel{\circ}{\mu}^{\prime}+1\right)-1=8 \pi r^{2} \int_{\mathbb{R}^{3}}\left(\frac{\tilde{x} \cdot v}{r}\right)^{2} \stackrel{\circ}{f}(r, v) \frac{d v}{\sqrt{1+v^{2}}}-4 \pi r^{2} e^{2 \grave{\lambda}^{\circ}} e^{2}
\end{aligned}
$$

We observe that, if $\stackrel{\circ}{f}$ is given and if we can solve (2.14)-(2.15) for $\stackrel{\circ}{\lambda}$ and $\stackrel{\circ}{e}$ then (2.16) determines at once $\stackrel{\circ}{\mu}$. Using the boundary condition (2.12) then determines $\stackrel{\circ}{\mu}$. So we can concentrate on (2.14)-(2.15). In what follows, we fix $f$ in (2.14)-(2.15) and we look for a unique global asymptotically flat solution $(\stackrel{\circ}{\lambda}, \stackrel{\circ}{e})$ of the system (2.14)-(2.15) above with regular centre. Note that, using 
the compact support of $\stackrel{\circ}{f}$ and the equations (2.14) and (2.15), it follows that $\stackrel{\circ}{\lambda}$ and $\stackrel{\circ}{e}$ tend to zero as $r \rightarrow \infty$. It also follows from (2.14) and (2.15) and the regularity of the solution that $\stackrel{\circ}{\lambda}^{\prime}(0)=0$ and $\stackrel{\circ}{e}(0)=0$.

\section{$3 \quad$ Existence of global solutions of the constraints}

In this section the existence of global solutions of the equations (2.14) and (2.15) will be proved. Let us state first of all the following result of [9] on which our global existence theorem relies:

Theorem 3.1 Let $V$ be a finite-dimensional real vector space, $N: V \rightarrow V$ a linear mapping, $G: I \times V \rightarrow V$ a smooth (i.e $C^{\infty}$ ) mapping and $g: I \rightarrow V$ a smooth mapping, where $I$ is an open interval in $\mathbb{R}$ containing zero. Consider the equation

$$
s \frac{d f}{d s}+N f=s G(s, f(s))+g(s)
$$

for a function $f$ defined on a neighborhood of 0 in $I$ and taking values in $V$. Suppose that each eigenvalue of $N$ has a positive real part. Then there exists an open interval $J$ with $0 \in J \subset I$ and a unique bounded $C^{1}$ function $f$ on $J \backslash\{0\}$ satisfying (3.1). Moreover $f$ extends to a $C^{\infty}$ solution of (3.1) on J. If $N, G$ and $g$ depend smoothly on a parameter $z$ and if the eigenvalues of $N$ are distinct then the solution also depends smoothly on $z$.

Proof. See Theorem 1 in [9], p.989.

Remark 1 The assumption that $N$ has distinct eigenvalues is to ensure that $N$ can be reduced to diagonal form by a similarity transformation depending smoothly on $z$. In particular, Theorem 3.1 applies if $N$ is already a diagonal matrix.

Theorem 3.2 (Local existence) Let $\stackrel{\circ}{f} \in C^{\infty}\left(\mathbb{R}^{6}\right)$ be nonnegative, compactly supported and spherically symmetric. Then, the equations 2.14 and 2.15) have a unique local and regular solution $(\stackrel{\circ}{\lambda}, \stackrel{\circ}{e})$ defined on some interval $[0, R]$, $R>0$. The solution depends smoothly on the parameter $q$.

Proof: Let $\stackrel{\circ}{f} \in C^{\infty}\left(\mathbb{R}^{6}\right)$ be nonnegative, compactly supported and spherically symmetric. By a regular solution we mean one which is smooth and for which $\stackrel{\circ}{\lambda}=0$ at $r=0$. It follows that for any regular solution $\stackrel{\circ}{\lambda}$ can be written in the form:

$$
\stackrel{\circ}{\lambda}(r)=r L(r)
$$

for a smooth function $L(r)$. Equation (3.2) implies $\stackrel{\circ}{\lambda}^{\prime}=L+r L^{\prime}$ and (2.14) (2.15) can be written:

$$
r L^{\prime}+L=\frac{1}{2 r}\left(1-e^{2 r L}\right)+4 \pi r e^{2 r L}\left(\int_{\mathbb{R}^{3}} \sqrt{1+v^{2}} f(r, v) d v+\frac{1}{2} e^{2 r L^{\circ}} e^{2}\right)
$$




$$
r \stackrel{\circ}{e}+2 \stackrel{\circ}{e}=-r \stackrel{\circ}{e}\left(L+r L^{\prime}\right)+r q \int_{\mathbb{R}^{3}} \stackrel{\circ}{f}(r, v) d v
$$

The function $e^{2 x}-1-2 x$ vanishes to first order at the origin and hence $e^{2 x}-$ $1-2 x=x^{2} F_{0}(x)$ for a smooth function $F_{0}$. Hence the equation for $L$ can be rewritten in the form

$$
r L^{\prime}+L=-L+\frac{r}{2} L^{2} F_{0}(r L)+4 \pi r e^{2 r L}\left(\int_{\mathbb{R}^{3}} \sqrt{1+v^{2}} f d v+\frac{1}{2} e^{2 r L_{e}^{\circ 2}}\right)
$$

Thus

$$
r L^{\prime}+2 L=r G_{1}(r, L, \stackrel{\circ}{e}, \stackrel{\circ}{f})
$$

where

$$
G_{1}(r, L, \stackrel{\circ}{e}, \stackrel{\circ}{f})=\frac{1}{2} L^{2} F_{0}(r L)+4 \pi e^{2 r L} \int_{\mathbb{R}^{3}} \sqrt{1+v^{2}} \stackrel{\circ}{f}(r, v) d v+2 \pi e^{4 r L^{\circ}} e^{2}
$$

and (3.4) reads, given (3.5):

$$
r \stackrel{\circ}{\circ}+2 \stackrel{\circ}{e}=-r \stackrel{\circ}{e}\left(-L+r G_{1}(r, L, \stackrel{\circ}{e}, \stackrel{\circ}{f})\right)+r q \int_{\mathbb{R}^{3}} \stackrel{\circ}{f}(r, v) d v .
$$

Hence:

$$
r \stackrel{\circ}{\circ^{\prime}}+2 \stackrel{\circ}{e}=r G_{2}(r, L, \stackrel{\circ}{e}, \stackrel{\circ}{f})
$$

where

$$
G_{2}(r, L, \stackrel{\circ}{e}, \stackrel{\circ}{f})=L \stackrel{\circ}{e}-r \stackrel{\circ}{e} G_{1}(r, L, \stackrel{\circ}{e}, \stackrel{\circ}{f})+q \int_{\mathbb{R}^{3}} \stackrel{\circ}{f}(r, v) d v
$$

Setting $G=\left(\begin{array}{l}G_{1} \\ G_{2}\end{array}\right)$ and $\Phi=\left(\begin{array}{c}L \\ 0 \\ e\end{array}\right)$ and using (3.5)-(3.6), the equations (2.14) and (2.15) can be written:

$$
r \frac{d \Phi}{d r}+2 \Phi=r G(r, \Phi(r))
$$

We apply Theorem 3.1 with $V=\mathbb{R}^{2}, N=\left(\begin{array}{ll}2 & 0 \\ 0 & 2\end{array}\right), N \Phi=2 \Phi$ to (3.7) and, since $G$ clearly depends smoothly on $q$, obtain the desired result. Thus Theorem 3.2 is proved.

Theorem 3.3 (Global existence, low charge) Let $\stackrel{\circ}{f} \in C^{\infty}\left(\mathbb{R}^{6}\right)$ be nonnegative, compactly supported and spherically symmetric with

$$
8 \pi \int_{0}^{r} s^{2}\left(\int_{\mathbb{R}^{3}} \sqrt{1+v^{2}} \stackrel{\circ}{f}(s, v) d v\right) d s<r .
$$

Then, for $q$ small enough, the equations 2.14) and (2.15) have a unique global and regular solution $(\stackrel{\circ}{\lambda}, \stackrel{\circ}{e})$ defined on $[0,+\infty[$ that satisfies the boundary condition $\stackrel{\circ}{\lambda}(0)=0$. 
Proof Let $\stackrel{\circ}{f} \in C^{\infty}\left(\mathbb{R}^{6}\right)$ be nonnegative, compactly supported and spherically symmetric. We assume that $f$ is fixed and satisfies (3.8). By Theorem 3.2, the equations (2.14) and (2.15) have a unique local regular solution on some interval $[0, R], R>0$. Again, Theorem 3.1 shows that, for fixed $\stackrel{\circ}{f}$, there exists $E>0$, such that for $q \in[-E, E], R$ can be chosen uniformly and the solution on $[0, R]$ depends continuously on the parameter $q$. Now, for fixed $\stackrel{\circ}{f}$ and $q$, the solution has a right maximal interval of existence $\left[0, R_{*}\left[, R_{*}=R_{*}(f, q)\right.\right.$. We have to show that $R_{*}=+\infty$. In fact, the second term in the r.h.s of (2.14) vanishes for $q=0$, as one can see by integrating (2.15) over [0,r], $r>0$. It follows that for $q=0,(2.14)$ and (2.15) have a global solution under the sole assumption (3.8) on $f$. Then by the stability theorem for ODE, for every $R>0$, there exists a number $E>0$, such that, for every $q \in[-E, E]$, the system (3.7) has a solution $\Phi_{E}$ that exists on $[0, R]$ (see Theorem 4 , p. 92 in $[6]$ ). Thus $R_{*}>R$. Now, we can choose $R$ large so that $\operatorname{supp} \stackrel{\circ}{f} \subset[0, R] \times \mathbb{R}^{3}$, i.e $\stackrel{\circ}{f}(r, v)=0$ for $r \geq R$. If $R_{0}$ is the radius of the support of the distribution function then $R$ may be chosen to be bigger than $m\left(R_{0}\right)+Q^{2} /\left(8 \pi R_{0}\right)$ for all $q$ in the interval $[E, E]$. Hence by the lemma of the appendix the solution extends to one which is global and regular. This completes the proof of the theorem.

Theorem 3.4 (Global existence, high charge) Let $\bar{f} \in C^{\infty}\left(\mathbb{R}^{6}\right)$ be nonnegative, compactly supported and spherically symmetric. Then, for $q$ large enough, the equations (2.14) and (2.15) have a global and regular solution $(\stackrel{\circ}{\lambda}, \stackrel{\circ}{e})$ defined on $[0,+\infty$ that satisfies the boundary condition $\stackrel{\circ}{\lambda}(0)=0$ for which $\stackrel{\circ}{f}$ is a constant multiple of $\bar{f}$. Moreover the charge to mass ratio $Q / M$ of the solution can be made as large as desired.

Proof We assume that $\bar{f}$ is fixed as in the assumptions of the theorem We set:

$$
\alpha=q^{-1} ; \quad \bar{f}=\alpha^{-k} \stackrel{\circ}{f} ; \quad \bar{e}=\alpha^{-(k-1)} \stackrel{\circ}{e}
$$

for some integer $k \geq 2$. Then (2.14)-(2.15) can be written as:

$$
\begin{gathered}
e^{-2 \grave{\lambda}}\left(2 r \stackrel{\circ}{\lambda}^{\prime}-1\right)+1=8 \pi r^{2}\left(\alpha^{k} \int_{\mathbb{R}^{3}} \bar{f}(r, v) d v+\frac{1}{2} e^{2 \lambda^{\lambda}} \alpha^{2(k-1)} \bar{e}^{2}\right) \\
2 \bar{e}+r \bar{e} \grave{\lambda}^{\prime}+r \bar{e}^{\prime}=-r \int_{\mathbb{R}^{3}} \bar{f}(r, v) d v .
\end{gathered}
$$

Introducing a variable $L$ as defined in (3.2) puts these equations into a form closely analogous to that obtained in the proof of the last theorem. In fact the left hand side has the same form as in that case. All that is changed is the form of the nonlinear terms on the right hand side. The equations depend on $\alpha$ as a parameter in a way which is smooth in a neighbourhood of $\alpha=0$. For $\alpha=0$ the function $\stackrel{\circ}{\lambda}$ vanishes identically while the equation for $\bar{e}$ becomes 
linear and has a global regular solution. From this point on we can argue just as in the proof of Theorem 3.3 to conclude that for $\alpha$ sufficiently small there is a unique global regular solution of these equations. Here we must use the fact that $m\left(R_{0}\right)+Q^{2} /\left(8 \pi R_{0}\right)$ is bounded independently of $\alpha$ for $\alpha$ small. The assumption that $\alpha$ is small corresponds to $q$ being large. The distribution function belonging to the solution is obtained from $f$ by a constant rescaling. The charge to mass ratio of the solution is proportional to $\alpha^{-k}$ and thus tends to infinity as $\alpha$ tends to zero. This completes the proof.

Remark 2 The solution in the exterior region is part of the Reissner-Nordström solution [5].

Remark 3 Our motivation in proving these theorems was to construct initial data for the Einstein-Vlasov-Maxwell system. The same arguments apply with other kinds of charged matter such as a charged fluid as sources for the Einstein equations.

Acknowledgments: One of us (ADR) thanks Curt Cutler for a helpful suggestion. This work was supported by a research grant from the VolkswagenStiftung, Federal Republic of Germany.

\section{A Appendix}

This appendix contains an analysis of exterior regions free of particles in initial data sets for the Einstein-Vlasov-Maxwell system. Let $\stackrel{\circ}{f}$ be an initial distribution function of compact support. Let $R_{0}$ be the radius of its support in space so that $\stackrel{\circ}{f}$ vanishes for all $r \geq R_{0}$. In this appendix we are only concerned with quantities on the initial hypersurface and so we will drop the label zero indicating the restriction of spacetime quantities to the initial hypersurface.

Lemma 1 Consider a solution of the constraint equations for the spherically symmetric Einstein-Vlasov-Maxwell system defined for $0 \leq r \leq R_{1}$ and having a regular centre. Suppose that radius $R_{0}$ of the support of the distribution function $f$ is less than $R_{1}$. Let $\tilde{M}=m\left(R_{0}\right)+Q^{2} /\left(8 \pi R_{0}\right)$. Then if $R_{1}>2 \tilde{M}$ the given solution extends to a unique solution of the constraints defined for all $r \in[0, \infty[$ which is asymptotically flat and has $f=0$ for $R \geq R_{0}$.

Proof Integrating the constraint equation (2.15) gives

$$
r^{2} e^{\lambda(r)} e(r)=q \int_{0}^{r} s^{2} e^{\lambda(s)} \int_{\mathbb{R}^{3}} f(s, v) d v
$$

For $r \geq R_{0}$ the upper limit $r$ in the integral can be replaced by $R_{0}$ or infinity without changing the value of the expression. It is equal to $Q / 4 \pi$ where $Q$ is 
the total charge of the system defined in Sect. 2. For $r \geq R_{0}$ the function $f$ vanishes and the mass function $m$ defined in Sect. 2 satisfies

$$
m^{\prime}=\frac{2 \pi}{r^{2}}(Q / 4 \pi)^{2}
$$

It follows that $\tilde{M}(r)=m(r)+Q^{2} /(8 \pi r)$ is independent of $r$. If the solution exists globally in $r$ and is asymptotically flat then taking the limit $r \rightarrow \infty$ shows that $\tilde{M}$ is equal to the ADM mass $M$. In any case $\tilde{M}$ is positive and it follows that in the exterior region $m=\tilde{M}-Q^{2} /(8 \pi r)$. In order to determine whether a solution can be extended to larger values of the radius it is enough to ensure that $1-2 \tilde{M} / r+Q^{2} /\left(4 \pi r^{2}\right)$ remains positive. For in that case we can define $\lambda$ by means of the relation

$$
e^{-2 \lambda}=1-2 \tilde{M} / r+Q^{2} /\left(4 \pi r^{2}\right)
$$

Note that $\lim _{r \rightarrow \infty} \lambda(r)=0$. Once this has been done we can define $\mu$ to be equal to $-\lambda$ and $e(r)=r^{-2} e^{-\lambda}(Q / 4 \pi)$ in the external region and this gives the unique solution satisfying the correct boundary conditions. If $r>2 \tilde{M}$ then $1-2 \tilde{M} / r+Q^{2} /\left(8 \pi r^{2}\right)$ is automatically positive and the desired result is obtained.

\section{References}

[1] Andréasson, H., The Einstein-Vlasov system / kinetic theory. Liv. Rev. Rel. 5, 7 (2002).

[2] Dafermos, M., Stability and instability of the Reissner-Nordström Cauchy horizon and the problem of uniqueness in general relativity. Preprint gr-qc/0209052

[3] Dafermos, M., Stability and instability of the Cauchy horizon for the spherically symmetric Einstein-Maxwell-scalar field equations. Preprint, to appear in Ann. Math.

[4] Friedrich, H. and Rendall, A. D., The Cauchy problem for the Einstein equations. In Schmidt, B. G. (ed.) Einstein's field equations and their physical applications. Lecture Notes in Physics 540. (Springer, Berlin, 2000).

[5] Hawking, S.W. and Ellis, G.F.R., The large-scale structure of space-time. (Cambridge University Press, Cambridge, 1973).

[6] Perko, L., Differential equations and dynamical systems. (Springer, New York, 2001).

[7] Rein, G. and Rendall, A.D., Global existence of solutions of the spherically symmetric Vlasov-Einstein system with small initial data. Commun. Math. Phys. 150, 561-583 (1992). 
[8] Rendall, A.D., The Einstein-Vlasov system. To appear in the proceedings of the workshop '50 years of the Cauchy problem in general relativity', Cargèse, 2002. Preprint gr-qc/0208082

[9] Rendall, A.D and Schmidt, B.G, Existence and properties of spherically symmetric static fluid bodies with a given equation of state. Class. Quantum Grav. 8, 985-1000 (1991). 